\title{
Documenter le futur: fantasmes, projections et decalages spatiotemporels
}

\author{
ALICE FORGE
}

Alice Forge, doutoranda em Artes Plásticas e Ciências da Arte na Universidade de Paris-1 Panthéon-Sorbonne. Nasceu em Paris em 1985 onde vive e trabalha. Artista plástica, mestre em Arte desde 2010 pela Universidade de Paris-1 Panthéon-Sorbonne. Desenvolve pesquisa de doutorado, sob a orientação do professor doutor Bernard Guelton, na linha de pesquisa "Ficções e Interações". Sua pesquisa se articula em torno da ideia de "documentar o futuro", uma proposta plástica desenvolvida ha três anos, onde a artista inventa uma comunidade de artistas fictícios do futuro, determinando o contexto político de sua atividade e realizando obras assinadas por eles. Forge utiliza um grande repertório de mídias para dar credibilidade à existência desses autores: instalação, fotografia, infografia, literatura, escultura, vídeo. Sua proposta é de criar uma coleção de autores e obras, que são apresentadas em exposições retrospectivas. Desse modo a artista opera os conceitos de arqueologia do futuro e de autoria, investigando a identidade do artista como um ato de disfarce e de construção fictícia. http://www.univ-paris1. $\mathrm{fr} /$ jp/recherche/page-perso/page/?tx_oxcspagepersonnel_pi1\%5Buid\%5D=e1701016053 


\section{- RÉSUMÉ}

Le concept de documentation future recèle un paradoxe : le mot documenter renvoie à des traces relevées dans un passé factuel, tandis que le futur ne peut, par définition, être qu'incertain, que l'objet de fantasmes, de projections. L'analyse suivante questionne ce paradoxe à travers l'analyse d'un motif cinématographique : un homme et une femme face à une tranche de séquoia, représentation symbolique du temps. Ce motif apparait dans trois œuvres majeures du cinéma : Vertigo, d'Alfred Hitchcock, La Jetée de Chris Marker, et l'Armée des douze singes, de Terry Gilliam. La répétition de ce motif est mise en rapport avec l'ouvrage de Pierre Bayard, Le plagiat par anticipation, afin de l'envisager sous l'angle du paradoxe temporel, plutôt que sous le régime de la chronologie historique. Cette analyse prend pour point de départ une œuvre intitulée Vozes Fantasmas, qui sur le principe de la documentation future propose d'écouter des murmures de spectateurs visitant une exposition qui n'a pas eu lieu.

\section{MOTS-CLÉS}

Documentation future, paradoxe temporel, vozes fantasmas.

\section{- RESUMO}

O conceito de documentação do futuro evoca um paradoxo: o verbo documentar refere-se a traços ou rastros preservados de um passado factual, enquanto o futuro por definição é algo incerto, objeto de especulações, fantasias e projeções. Nossa reflexão questiona este paradoxo a partir da análise de uma cena cinematográfica: um homem e uma mulher em frente de um corte de sequoia, que é a representação simbólica do tempo. Esta cena aprece em três grandes obras do cinema: Vertigo (Um corpo que cai) de Alfred Hitchcock, La Jetée de Chris Marker, e Os Doze Macacos de Terry Gilliam. A repetição desta cena é abordada em relação à obra de Pierre Bayard, Plágio por antecipação, a fim de considerá-la em termos de paradoxo temporal, e não como uma cronologia histórica. Esta análise tem como ponto de partida um trabalho artístico intitulado Vozes Fantasmas, que seguindo o princípio de documentação do futuro propõe ouvir os sussurros de espetadores durante uma exposição que ainda não aconteceu.

- palaVRas-chaVe

Documentação do futuro, paradoxo temporal, vozes fantasmas.

Ils sont sans souvenirs, sans projets. Leur temps se construit simplement autour d'eux, avec pour seul repère le goût du moment qu'ils vivent.

La Jetée, Chris Marker

Le concept de documentation future recèle un paradoxe : le mot documenter renvoie à des traces relevées dans un passé factuel, tandis que le futur ne peut, par définition, être qu'incertain, que l'objet de fantasmes, de projections.

Artistiquement, il s'agit de rendre compte, à travers la fiction, d'un fantasme ou d'une projection, en leur conférant une esthétique documentaire, une valeur d'archive.

L'œuvre proposée pour cette exposition, Voix Fantômes, opère dans cet esprit d'archivage : elle consiste en l'enregistrement de dialogues de spectateurs, visitant une exposition qui n'a pas encore eu lieu. En français, le mot Fantôme est issu de la même racine que Fantasme. Le contenu de cette exposition fantasmée donne lieu à des commentaires, qui sont, pour le spectateur de l'exposition d'Uberlândia, la seule voie d'accès à des œuvres qui ne lui sont pas encore présentées. Quels sont les enjeux esthétiques de la distorsion temporelle mise en œuvre dans ce travail, et dans le concept même de documentation future? 
Pour déterminer ces enjeux, je vais étudier un motif qui apparait dans trois films traitant de la hantise et de la disparition : Vertigo, d'Alfred Hitchcock ${ }^{1}$, adapté de Boileau et Narcejac ${ }^{2}$, La Jetée, de Chris Marker ${ }^{3}$, et le film de Terry Gilliam qui s'inspire de La Jetée, L'Armée des Douze Singes ${ }^{4}$. Ce motif symbolique est celui d'une tranche de séquoia géant, représentation contenue en un unique objet de plusieurs strates de temps. II constitue un repère temporel et une matérialisation d'un déploiement du temps en cercles concentriques, chacun des âges de l'arbre est visible simultanément. Ce motif qui lie ces trois œuvres sera mis en rapport avec le concept de plagiat par anticipation, développé par Pierre Bayard ${ }^{5}$, concept qui relève lui aussi du paradoxe temporel. Nous essaierons de montrer qu'il serait réducteur de ne considérer ces multiples passages d'un film à l'autre comme de simple citations des films antérieurs, mais qu'il s'agit bien d'un dialogue entre les fantômes passés et les possibles surgissements futurs.

\section{Vertigo (Sueurs froides)}

It's too late.

Il est trop tard.

Vertigo, d'Alfred Hitchcock

Vertigo est le premier, chronologiquement, de ces trois films qui mettent en scène la coupe de séquoia. II relate l'histoire d'un homme, Scottie, qui est engagé pour veiller sur Madeleine, la femme d'un vieil ami, Gavin Elster. Son mari la croit possédée par le fantôme d'une ancêtre suicidaire, Carlotta Valdès. II prend Madeleine en filature, lors de longues scènes où il l'épie de loin. Madeleine, figure inaccessible, troublante, au comportement mélancolique et étrange, se rend au musée, y contemple le portrait de Carlotta Valdès, porte un bouquet sur sa tombe, et répète inlassablement ces trajets avec Scottie sur ses talons. II tombe éperdument amoureux d'elle. Un jour il la suit jusqu'au Golden Gate Park près des piliers que Gavin Elster appelle les " portails du passé », dans une phrase assez énigmatique. Ce lieu désert devient un lieu de passage dans le temps, propice à Carlotta qui semble prendre possession de Madeleine : Elle se jette à l'eau et Scottie la sauve. Ils se rapprochent.

Lors d'une balade avec Madeleine dans le parc de Muir Woods, près de San Francisco, leur marche les conduit devant la tranche d'un séquoia géant, où sont affichées les dates d'évènements marquants de l'histoire des États-Unis. Madeleine hantée par le fantôme de Carlotta Valdès, tend son bras ganté de noir et désigne deux cercles de croissance de l'arbre, et prononce cette phrase énigmatique : « Ici je suis née, ici je suis morte, ça n'aura été qu'un moment pour toi, tu ne l'as même pas remarqué ». Le paradoxe temporel qui existe soudain entre ce geste et cette parole, actions d'une femme vivante, et ce symbole noueux d'un temps révolu, fait apparaître symboliquement au spectateur du film le fantôme troublant d'une femme disparue. Madeleine est une pâle figure dans ces bois sombres, auxquels les troncs séculaires

\footnotetext{
HITCHCOCK, A. Sueurs Foides (Vertigo), 127 min, 1958.

BOILEAU et NARCEJAC. Sueurs froides. Éditions. Gallimard col. Folio Policier, 1954.

MARKER, CH. La Jetée, 28 min, 1962.

GILLIAM, T. L’Armée des Douze Singes (Twelve Monkeys) 129 min, 1995.

BAYARD, P. Le Plagiat par Anticipation, Éditions de Minuit, col. Paradoxe, 2009, 160 p.
} 
des séquoias donnent une atmosphère presque mythologique. Plusieurs strates de temps se confondent à cet instant, on ne sait plus qui, de Carlotta ou de Madeleine prononce ces propos étranges et infiniment tristes.

Plus tard, Madeleine sera poussée au suicide par le fantôme de Carlotta, et se jettera d'un clocher. Scottie, sujet à de très violentes crises de vertige, sera incapable de la sauver. Après des mois d'un deuil manifestement impossible, Scottie croisera une femme, Judy, dont la ressemblance avec Madeleine le fascinera. II parviendra lentement à la métamorphoser pour faire revivre l'image de son amante morte. Judy, rousse et vive, est en fait la femme que Gavin Elster, le mari de Madeleine, avait engagée pour tromper Scottie, et lui faire croire à la folie de Madeleine, ou au fantôme de Carlotta ; Gavin Elster l'avait engagée pour qu'elle fasse de Scottie le témoin d'un suicide qui était en réalité une mise en scène destinée à maquiller le meurtre de la véritable Madeleine, jetée du clocher par Gavin Elster. Judy, qui s'est elle aussi éprise de Scottie, alors qu'elle jouait le rôle de Madeleine, accepte de rejouer à contrecœur le jeu qu'il lui impose, à savoir de redevenir Madeleine. La vérité éclate, Scottie comprend qu'il a été dupé, et dans une confrontation avec Judy dans le clocher où avait eu lieu le meurtre, Judy chute et Scottie perd, une deuxième fois, la femme fantôme-fantasme qu'il a aimé et poursuivie.

\section{La Jetée}

Le temps s'enroule à nouveau. L'instant repasse. La Jetée, Chris Marker

Chris Marker reprend, quatre ans plus tard, le motif du séquoia dans son « ciné-roman » La Jetée, moyen métrage entièrement construit autour de l'idée de paradoxe temporel et de hantise, et constitué presque exclusivement de clichés fixes en noir et blanc. La narration est assurée par une voix-off, qui peut se rapprocher de l'esthétique narrative que je tente d'obtenir dans mon travail, avec les voix fantômes.

L'histoire diffère beaucoup de l'intrigue de Vertigo. Néanmoins le film commence sur cette phrase : «Ceci est l'histoire d'un homme marqué par une image d'enfance ». L'image d'enfance dont il est question est une scène dont il a été témoin sur la jetée d'un aéroport : un homme est tué devant les yeux d'une femme. Mais c'est peut être le visage de cette femme qui s'est imprimé le plus profondément dans la mémoire de cet enfant. Comme dans Vertigo, c'est le souvenir d'une femme perdue, fantasmée, disparue qui sert d'accroche au voyage dans le temps : Scottie aura cherché à ressusciter le passé en transformant le présent et Judy, tandis que le héros de La Jetée voyage dans ses propres souvenirs pour tenter de retrouver cette femme dont l'image l'obsède. Le couple de La Jetée est, d'une certaine manière, un reflet déformé de celui de Vertigo.

L'histoire de La Jetée est la suivante : dans un futur post-apocalyptique, après la troisième guerre mondiale, des survivants terrés sous la surface de Paris s'efforcent d'envoyer dans le passé des voyageurs, pour acquérir des vivres, des médicaments. Beaucoup de ces tentatives se soldent par des échecs, beaucoup des cobayes ne survivent pas à ces expériences de voyage dans le temps. Un homme, dont on ne connaîtra jamais le nom, y parvient, parce qu'il a dans son passé un souvenir très fort, celui d'un visage de femme « seul souvenir du temps de paix à avoir traversé le temps de guerre ». Ce visage, aperçu enfant sur la jetée d'Orly, est l'accroche nécessaire 
pour favoriser son retour dans le passé.

L'homme rencontre cette femme, vouée à disparaitre, dans ce conflit qui menace le monde de paix qu'il visite. Ensemble, ils parcourent la ville, dans une intimité de l'instant présent. II vient la visiter, par épisodes. « Elle l'appelle son spectre ». II ne situe pas ses allers et retours dans le temps, le temps n'a plus de trame, plus de continuité. Mais c'est elle, la femme qui hante ses souvenirs, la femme qui doit mourir dans un conflit qui détruira le monde, c'est elle le fantôme qu'il poursuit, comme Scottie poursuit désespérément l'image de Madeleine, jusqu'à modeler l'apparence de Judy afin qu'elle coïncide avec celle de l'amour perdu. II semble toujours revenir vers elle. Elle ne le fuit pas, mais l'issue fatale de leur amour est évidente.

Ils se retrouvent, lors d'un de ces épisodes, au jardin des plantes, devant la coupe d'un séquoia. Cette scène, que Chris Marker désignera dans Sans Soleil ${ }^{6} \mathrm{com}$ me une référence à son film favori, Vertigo, déforme et transforme le motif originel d'Hitchcock : L'homme désigne un point en dehors du séquoia, et « s'entend dire, comme en rêve » à sa compagne qu'il vient de «quelque part par là ». II se situe lui même hors du temps, hors de la chronologie naturelle. La femme porte un chignon qui fait écho à celui de la Madeleine d'Hitchcock : les cheveux enroulés en spirale qui ne sont pas sans rappeler, à leur tour, les cercles concentriques du séquoia.

Le film s'achève sur la Jetée d'Orly, là où coïncide le passé de l'homme - son souvenir d'enfance - et son présent tragique : Il est l'homme qui meurt sous les yeux de son amante, et sous ses propres yeux d'enfant. Le paradoxe temporel est intense: ce qui permet à l'homme de se projeter dans le passé est le souvenir de sa propre mort. Le titre du film de Marker, La Jetée, désigne à la fois le lieu où se joue ce paradoxe temporel, lieu symbolique d'où partent des avions vers l'ailleurs et l'idée de déplacement, de fuite, de projection dans le temps : Le héros de La Jetée est lancé vers son propre destin, sans possibilité d'y échapper.

\section{Twelve monkeys (L’armée des douze singes)}

The Future is History.

Le futur est de l'histoire ancienne.

Lu sur l'affiche de Twelve Monkeys, de Terry Gilliam

Twelve Monkeys, réalisé en 1995 par Terry Gilliam, reprend le synopsis de La Jetée. Gilliam a affirmé n'avoir pas vu le film de Chris Marker, de peur d'être trop influencé?.

L'histoire est sensiblement la même : Un homme marqué par une scène d'enfance - par le visage d'une femme - est envoyé dans le passé, suite à la mort de $99 \%$ de la population mondiale.

Mais elle diverge sur plusieurs points: ce n'est pas, comme dans La Jetée, une guerre nucléaire qui a anéanti la majeure partie de l'humanité, mais l'œuvre d'un seul homme, qui a répandu un virus mortel à dessein. James Cole n'est donc pas à la recherche, comme dans La Jetée, de moyens de survie mais tente d'empêcher la fin du monde. Les allers et retours dans le passé du héros, James Cole, sont plus aléatoires

\footnotetext{
6 MARKER, CH. Sans Soleil, 100 min, 1983.

7 Dossier pédagogique de Jean Douchet sur L’Armée des Douze Singes, BIFI. 
et plus complexes ; ce dernier ne revient pas systématiquement vers la femme dont le visage l'obsède : il ne la reconnait pas immédiatement. II semble qu'il voyage littéralement dans le temps, et non, comme dans La Jetée, dans son propre passé. C'est un film de science-fiction d'une façon plus affirmée que ne l'est La Jetée, c'est-à-dire qu'il utilise les codes littéraires du genre. C'est aussi, avant tout, un film paranoïaque, mettant en scène des fous : le doute est maintes fois posé que James Cole ne soit qu'un malade mental qui prophétise la fin du monde.

La femme qui l'accompagne, Kathryn Railly, reflet du personnage féminin passif de La Jetée, est sa psychiatre, et une spécialiste du syndrome de Cassandre, dont sont atteints les fous prophètes. La formule, que l'on doit à Gaston Bachelard, s'appuie sur le personnage mythologique de Cassandre, frappée de malédiction : elle avait le don de voir l'avenir mais était incapable de faire accepter la validité de ses prophéties. C'est Kathryn qui met James en doute, le fait interner, puis finit par le croire. Ils deviennent des fugitifs, considérés comme fous et dangereux par la société.

Dans une scène où tous les deux sont poursuivis par les autorités, ils se cachent dans un cinéma et se déguisent pour passer inaperçus. La scène qui est projetée, à ce moment, est précisément la scène du séquoia issue de Vertigo. James Cole semble bouleversé et dit à Kathryn qu'il se souvient d'avoir vu ce film lorsqu'il était enfant. II ajoute : “c'est comme ce qui nous arrive. C'est comme le passé. Le film ne change pas, il ne peut pas changer, mais chaque fois qu'on le regarde il semble différent, parce que nous sommes différents ». Ils sortent de la salle obscure et se retrouvent dans la lumière, déguisés : c'est à ce moment que James Cole reconnait en Kathryn la femme dont il a toujours rêvé, c'est-à-dire dont il s'est toujours souvenu. Kathryn porte une perruque blonde, elle devient un fantôme cinématographique de la Madeleine d'Hitchcock, la Judy rousse qui se métamorphose en Madeleine blonde. Ces deux moments de Vertigo, la scène devant la coupe du séquoia, d'une part, où Madeleine se confond avec le fantôme de Carlotta Valdès, et, d'autre part le moment ou Judy se transforme en Madeleine, et ressuscite ainsi l'amour mort de Scottie, sont condensés en un seul moment dans Twelve Monkeys : Le cinéma est la mémoire des bois obscurs dans lesquels se déroule la scène de Vertigo, et lorsque James et Kathryn en sortent, le hall de ce cinéma est la chambre d'hôtel dans laquelle Judy devient la femme disparue. Kathryn se transforme soudain en l'image du passé que Cole poursuivait.

Mais, particularité de Twelve Monkeys, Kathryn est, à cet instant là, également troublée par la vision de James déguisé, différent. Elle lui dit qu'elle a toujours eu l'impression de le connaître, qu'elle se souvient de lui habillé et maquillé ainsi. La projection et le voyage dans le passé se confondent avec une vision prophétique du futur : Kathryn n'est pas seulement le fantôme désiré, elle est aussi la prophétesse, la Cassandre qu'elle étudiait auparavant, celle qui prédit l'avenir mais est condamnée à ne pas être crue. Plusieurs strates de temps coïncident et coexistent à ce moment, souvenir, fantasme, anticipation de l'inévitable.

Car l'issue de Twelve Monkeys est, à ce moment, évidente: Cole va mourir devant Kathryn, et devant l'enfant qu'il était. La scène obsessionnelle de sa mort, souvenir, rêve ou prophétie, nous a déjà été présentée auparavant dans le film. Nous reconnaissons aussi James et Kathryn pour ses protagonistes. Mais dans cette version sensiblement différente, la scène de l'assassinat de James est filmée au ralenti, et Kathryn, après avoir pleuré son amant, cherche des yeux l'enfant, qu'elle sait être présent, et lui adresse un regard plein d'espoir et de tendresse. 


\section{Fictions transfuges \& le plagiat par anticipation : hantise intertemporelle}

Cette manière qu'ont les morts de nous hanter par l'ombre de leur faits et gestes signifie que la fiction d'un être est interminable, qu'elle ne cesse pas avec lui ${ }^{8}$.

La fiction biographique, Daniel Oster

Ce cas de passages d'une fiction à une autre appelle naturellement à un rapprochement avec le concept développé par Richard Saint-Gelais, la transfictionnalité. «Par 'transfictionnalité', j'entends le phénomène par lequel au moins deux textes, du même auteur ou non, se rapportent conjointement à une même fiction, que ce soit par la reprise de personnages, prolongement d'une intrigue préalable ou partage d'univers fictionnel ${ }^{9}$ ". Dans chacun des cas cinématographiques que nous avons préalablement étudiés, la référence à des films précédents est claire et assumée comme telle, il semble qu'elle ne relève pas tout à fait d'un cas de transfictionnalité : Si les couples qui évoluent dans chacun de ces films se ressemblent et se répondent, on ne peut parler d'identité ; Les intrigues de Vertigo, de La Jetée et de Twelve Monkeys ne prétendent pas se prolonger ; L'univers fictionnel qui sert de théâtre à ces intrigues diffère dans chacun des films ; même dans le cas de La Jetée et de Twelve Monkeys, alors que le synopsis de l'un sert de support à l'autre, la vision apocalyptique diffère énormément. Je n'ai pas ici la prétention de circonscrire la définition de la transfictionnalité, telle que la définit Saint-Gelais dans son ouvrage encyclopédique et extrêmement détaillé. Néanmoins, je préfèrerais employer le terme de hantise intertemporelle, qui me semble mieux définir le phénomène trouble de surimpression qui unit ces trois œuvres.

Afin de développer cette idée, je vais faire appel à un autre ouvrage ; Pierre Bayard développe, dans son livre Le Plagiat par Anticipation, une théorie qui semble peu défendable au premier abord : des auteurs antérieurs se seraient inspiré ou auraient copié des auteurs à venir ${ }^{10}$. Voltaire aurait ainsi été influencé par Conan Doyle, et Kafka se serait inspiré de l'imaginaire des régimes totalitaires, tandis que Maupassant aurait plagié Proust. Pierre Bayard nous propose un paradoxe temporel au regard de l'Histoire, mais qu'il défend habilement en montrant que l'histoire littéraire, I'histoire des idées, n'a de repère temporel que le lecteur, dont la culture et le regard est composé de strates de temps qui sont, à la manière du séquoia de Vertigo, toutes présentes dans un même lieu et un même moment. II met en lumière la plasticité du temps en montrant qu'en matière de culture, la chronologie linéaire n'a pas court, car elle est moins pertinente que les rapports de cohérence intertextuels que le lecteur fabrique de lui-même. " De même qu'il est impossible de savoir ce que serait le texte de Maupassant si Proust n'avait pas existé, il est impossible de savoir ce que serait la lecture de Tristan et Iseult pour quelqu'un qui n'aurait pas connu l'imaginaire Romantique $^{11} \gg$. Pierre Bayard pose, dans cet ouvrage, des questions légitimes, sous couvert d'une position qui semble, au premier abord, absurde.

Les connexions que nous pouvons établir entre Vertigo, La Jetée et Twelve Mon-

\footnotetext{
8 OSTER, D. « La fiction biographique », dans Encyclopædia Universalis, supplément Universalia, 1992, p. 404

9 SAINT-GELAIS, R. Fictions Transfuges, la transfictionnalité et ses enjeux. Éditions. Du Seuil, coll. Poétique, 2011. p. 607.

${ }^{10}$ BAYARD, P. Le Plagiat par Anticipation. Éditions de Minuit, col. "Paradoxe", 2009. p.160.

11 BAYARD, P. Le Plagiat par Anticipation, p.56.
} 
keys, relèvent d'un champ complexe de références croisées et de résonnances, qu'il serait réducteur de ne considérer que dans une logique chronologique. En effet, regarder ces trois films enrichit chacun d'entre eux, et montre les lectures potentielles qui se trouvent en germes dans le film le plus ancien des trois, Vertigo. Ainsi, ces trois œuvres qui parlent de hantise, et de disparition ne sont pas des produits successifs, mais forment un réseau de plusieurs temporalités qui se nouent, s'enracinent les unes dans les autres. Chacun de ces films hante, d'une certaine façon, les deux autres.

Dans mon œuvre Voix Fantômes, et dans mon travail plastique de manière général, je suis moi-même hantée par cette image du séquoia, dont je ne sais plus vraiment si elle m'a d'abord marquée dans Twelve Monkeys, dans La Jetée ou dans Vertigo. L'origine du motif à moins d'importance, dans mes travaux, que sa persistance dans ma mémoire, et sa hantise est appuyée par sa répétition, sa transformation, la pluralité d'images et de sentiments qui s'y accrochent et s'y enracinent. Le visage de Kim Novak dans Vertigo est, lui-même, le fantôme d'un passé qui, à travers le cinéma, se convoque à l'envi, se conjugue avec ses fantômes futurs, jusqu'à s'y confondre. Immortelle, elle est un fantôme intemporel. Sa voix répète infiniment « it's too late, it's too late » et chaque fois que ma fascination me pousse à revoir ce passage, je pense aussi à la phrase de Marker dans la Jetée, cette phrase qui concentre tout le pouvoir du cinéma : «le temps s'enroule à nouveau, l'instant repasse ».

Mon propre traitement du temps et de sa distorsion, ma volonté de faire coexister dans un seul espace des voix, archives du passé, avec des fantasmes futurs est directement liée à cette collection d'images qui se déploient et se rassemblent à la fois, autour de cet objet symbolique : la coupe du séquoia. C'est pourquoi je le figure, dans mon œuvre, en faisant référence à la spirale de Vertigo : spirale du chignon de Madeleine, spirale de l'escalier vertigineux du clocher, spirale du séquoia. Car il est bien question de vertige, le vertige que provoque tout paradoxe, en mettant en rapport des choses qui, selon une logique naturelle, ne devraient pas coexister.

Dans Twelve Monkeys, James Cole écoute, en 2035, l'enregistrement d'une voix déformée, abîmée, une voix d'archive, qui s'avère être l'enregistrement d'un message téléphonique laissée par Kathryn plus tard - c'est-à-dire plus tard dans le récit, mais plus tôt dans la chronologie historique des évènements, en 1996. La confusion, le télescopage de ces deux réalités créent un paradoxe. Ce message téléphonique d'outre-tombe, car c'est bien de cela qu'il s'agit, fait écho à la voix presque éteinte de Madeleine, lorsque devant la coupe de séquoia elle profère des paroles absurdes pour une vivante, qui ne peuvent avoir de sens que si c'est le fantôme de Carlotta Valdès qui parle à travers elle. C'est l'effet de trouble que je cherche à obtenir en faisant entendre au spectateur des voix enregistrées, celle d'un couple qui pourrait, implicitement, rappeler les couples cinématographiques de ces trois films. Ces voix sont des archives nécessairement capturées dans le passé, qui évoquent un futur hypothétique et sont entendues dans le présent. Le seul repère temporel est ce dialogue de voix, le plaisir et l'ironie avec lesquels ils commentent les œuvres. Cette distorsion du temps rappelle la belle phrase issue de la Jetée : « lls sont sans souvenirs, sans projets, leur temps se construit simplement autour d'eux, avec pour seul repère le goût du moment qu'ils vivent ». 


\section{Références}

BAYARD, Pierre. Le Plagiat par anticipation. Éditions de Minuit, col. «Paradoxe», 2009.

BOILEAU et NARCEJAC. Sueurs froides. Éditions Gallimard col. Folio Policier, 1954.

SAINT-GELAIS, Richard. Fictions transfuges, la transfictionnalité et ses enjeux. Éditions du Seuil, coll. Poétique, 2011.

Notices

Oster, Daniel. La fiction biographique, dans Encyclopædia Universalis, supplément Universalia, 1992.

Filmographie

HITCHCOCK, Alfred. Sueurs Foides (Vertigo), 127 min, 1958.

MARKER, Chris. La Jetée, 28 min, 1962.

MARKER, Chris. Sans Soleil, $100 \mathrm{~min}, 1983$.

GILLIAM, Terry. L’Armée des Douze Singes (Twelve Monkeys) 129 min, 1995. 